\title{
The relaxation of the second moments in rapid shear flows of smooth disks
}

\author{
M.Y. Louge a, J.T. Jenkins ${ }^{b}$ and M.A. Hopkins ${ }^{c}$
}

a Sibley School of Mechanical and Aerospace Engineering, Cornell University

b Department of Theoretical and Applied Mechanics, Cornell University, Ithaca, NY 14853

c Ice Engineering Research, US Army Cold Regions Research and Engineering Laboratory, Hanover, NH 03755

\section{Proceedings of the $2^{\text {nd }}$ US/Japan Conference on Micromechanics of Granular Materials, Clarkson University, August 1991.}

\begin{abstract}
This paper compares the results of numerical simulations for two-dimensional, rapid, homogeneous shear flows of identical, smooth, inelastic disks with the predictions of Jenkins and Richman [JFM 192, 313-328 (1988)] for the relaxation of the second moments of the velocity distribution function following a homogeneous, but anisotropic disturbance of their steady values. For nearly elastic disks, the time-history of the relaxation is in excellent agreement with the theory in both its dense and dilute limits. However, deviations are observed in the case of inelastic particles.
\end{abstract}

\section{Discussion}

Jenkins and Richman [1] consider rapid granular flows of identical, smooth, inelastic disks of mass $\mathrm{m}$, diamete: $\sigma$, and coefficient of restitution $e$. They introduce an anisotropic Maxwellian velocity distribution function based on the full second moment of the velocity distribution function $\mathbf{K} \equiv<\mathbf{C C}>$, and determine approximate analytic solutions of the balance law for $\mathbf{K}$ for steady, simple shear of both dilute and dense systems with arbitrary values of $e$. For general flows they write this balance law as

$$
\rho \dot{K}_{\alpha \beta}+\frac{\partial Q_{y \alpha \beta}}{\partial x_{\gamma}}+P_{\mu \beta} \frac{\partial u_{\alpha}}{\partial x_{\mu}}+P_{\mu \alpha} \frac{\partial u_{\beta}}{\partial x_{\mu}}-m \chi\left[C_{\alpha} C_{\beta}\right]=0
$$

where $\rho$ is the mean mass density, the overdot denotes a time derivative following the mean flow, the Greek indices take the values 1 and 2, and

$P_{\alpha \beta} \equiv \rho K_{\alpha \beta}+\Theta_{\alpha \beta}$

$$
\text { AC22-88PC } 88947
$$


is the stress tensor expressed here as the sum of streaming and collisional components, respectively. The flux $Q_{\text {pa }} \beta$ of second moment also includes streaming and collisional contributions; however, in homogeneous flows, the divergence of this flux vanishes. The last term is a collisional source of second moment. Jenkins and Richman [1] express it as a sum of three integrals

$m \chi\left[C_{\alpha} C_{\beta}\right]=A_{\alpha \beta}+\hat{E}_{\alpha \beta}+\hat{G}_{\alpha \beta}$,

which they calculate along with $\Theta_{\alpha \beta}$ in the dilute and dense limit. In the steady simple shear that we consider, the mean velocity is directed along the $\mathrm{x}_{1}$ axis of a cartesian coordinate system and varies linearly with the $x_{2}$ coordinate. For convenience, we express Eq. (1) in dimensionless form. To do this, we take the reference time and distance to be, respectively, the inverse of the shear rate and the particle diameter. Dimensionless quantities are denoted by a tilde ( $\sim$ ).

In the dilute limit, the area fraction $v$ of the particles is small and $\Theta_{\alpha \beta}=\hat{G}_{\alpha \beta}=0$. Using Eqs. (60)-(61) of Jenkins and Richman for $A_{\alpha \beta}$ and $\hat{E}_{\alpha \beta}$, Eq.(1) becomes $\frac{\partial \tilde{\mathrm{K}}_{11}}{\partial \tilde{\mathrm{t}}}+2 \tilde{\mathrm{K}}_{21}=-\frac{2 \mathrm{G}(1+\mathrm{e})}{\pi^{3 / 2}}\left\{\left[1+\frac{3}{4}(1-\mathrm{e})\right] \tilde{\mathrm{T}}^{1 / 2} \mathrm{I}(\alpha)\left(\tilde{\mathrm{K}}_{11}-\tilde{\mathrm{K}}_{22}\right)+(1-\mathrm{e}) \tilde{\mathrm{T}}^{3 / 2} \gamma(\alpha)\right\}$

$\underline{\partial \tilde{\mathrm{K}}} . \underline{.22}$ $\partial \tilde{\mathrm{t}}$

$$
=+\frac{2 G(1+e)}{\pi^{3 / 2}}\left\{\left[1+\frac{3}{4}(1-e)\right] \tilde{T}^{1 / 2} I(\alpha)\left(\tilde{K}_{11}-\tilde{K}_{22}\right)-(1-e) \tilde{T}^{3 / 2} \gamma(\alpha)\right\}
$$

and

$$
\frac{\partial \tilde{\mathrm{K}}_{21}}{\partial \tilde{\mathrm{t}}}+\tilde{\mathrm{K}}_{22}=-\frac{4 \mathrm{G}(1+\mathrm{e})}{\pi^{3 / 2}}\left[1+\frac{3}{4}(1-\mathrm{e})\right] \tilde{\mathrm{T}}^{1 / 2} \mathrm{I}(\alpha) \tilde{\mathrm{K}}_{21},
$$

where $G \equiv v(16-7 v) / 16(1-v)^{2}, \tilde{T} \equiv\left(\tilde{K}_{11}+\tilde{K}_{22}\right) / 2$ is the granular temperature, and $\alpha \equiv\left[\left(\tilde{\mathrm{K}}_{11}-\tilde{\mathrm{K}}_{22}\right)^{2}+4 \tilde{\mathrm{K}}_{21}\right]^{1 / 2} / 2 \tilde{\mathrm{T}}$.

The functions $\gamma(\alpha)$ and $I(\alpha)$ are given by the integrals

$I(\alpha) \equiv \int_{0}^{2 \pi} \sin ^{2} 2 \theta(1-\alpha \cos 2 \theta)^{1 / 2} \mathrm{~d} \theta$,

and

$\gamma(\alpha) \equiv \int_{0}^{2 \pi}(1-\alpha \cos 2 \theta)^{3 / 2} \mathrm{~d} \theta$ 
In this work, they are evaluated numerically.

In the dense limit, $P_{\alpha \beta}=\Theta_{\alpha \beta}$. We employ Eq. (79) of Jenkins and Richman for $\Theta_{\alpha \beta}$ and their Eqs. (76)-(78) for $A_{\alpha \beta}, \hat{E}_{\alpha \beta}$ and $\hat{G}_{\alpha \beta}$. Then Eq.(1) becomes

$\frac{\partial \tilde{T}}{\partial \tilde{t}}=-\frac{G(1+e) \tilde{T}^{1 / 2}}{\pi^{3 / 2}}[2(1-e) \tilde{T} \gamma(\alpha, R)-S(\alpha, R) / 8]$,

and

$\frac{\partial \tilde{\mathrm{K}}_{21}}{\partial \tilde{\mathrm{t}}}=-\frac{\mathrm{G}(1+\mathrm{e}) \tilde{\mathrm{T}}}{\pi^{3 / 2}}[\mathrm{~N}(\alpha, \mathrm{R})+(1-\mathrm{e}) \mathrm{H}(\alpha, \mathrm{R}) / 4-\mathrm{J}(\alpha, \mathrm{R})-\mathrm{I}(\alpha, \mathrm{R}) / 2]$,

where $R \equiv 1 / 8 \tilde{T}^{1 / 2}$. In Eqs. (A17)-(A22) of an Appendix, Jenkins and Richman [1] provide approximate expressions for the integrals $\gamma, \mathrm{S}, \mathrm{N}, \mathrm{H}, \mathrm{J}$ and $\mathrm{I}$ in terms of $\mathrm{R}$ and $a \equiv \alpha / 2 \pi^{1 / 2} R$. Because in the lowest order theory for the dense limit it is assumed that $K_{\alpha \beta}$ and the symmetric part of $\partial u_{\alpha} / \partial x_{\beta}$ have the same eigenvectors, the theory in its present form does not permit the independent variations of $\mathrm{K}_{11}$ and $\mathrm{K}_{22}$ that are possible in the simulation.

The numerical simulations are carried out using an algorithm described by Hopkins and Louge [2]. In this scheme, thu flow domain is periodic in the streamwise direction. Periodic images of each disk above and below the square central domain of width $\mathrm{L}$ are used to maintain the constant mean shear rate, without affecting the statistics of any higher moment of the velocity field. After a given time step, the algorithm calculates the position of each particle from the previous position and velocity at the beginning of the interval. Collisions occur when a particle overlaps slightly with a neighbor. The velocities of the particles emerging from a collision are calculated by considering the balance of linear momentum in the collision. The coefficient of resatution characterizes the incomplete restitution of translational velocity in the direction parallel to the line of centers. A constant simulation time step is chosen so the average overlap does not exceed $0.5 \%$ of the particle diameter during each run.

At the onset of a simulation, the required number of identical disks is placed in the periodic cell with a mean velocity corresponding to their location in the shear field and a small random fluctuating component to initiate collisions. The disk velocities are adjusted to cancel the net momentum of the system, and the initial position of the disks is such that the center of mass of the system lies at the center of the periodic domain. First, a typical simulation is carried out for a dimensionless time $\mathrm{t}^{\dagger}=100$ designed to create a random configuration independent of the initial state of the system. Then an artificial imbalance in 
the second moments is produced by rescaling instantaneously the fluctuating velocity $C_{\alpha}^{0}(j)$ of each paricle of index $\mathrm{j}$ in the periodic domain to the new value

$C_{\alpha}^{l}(j)=\xi(\alpha) C_{\alpha}^{0}(j)$

where $\xi(\alpha)$ is a constant that need not be the same for the two components of the

fluctuating velocity. While they relax back to their steady values, the seconds moments are computed at regular time intervals by averaging over the $\mathrm{J}$ particles of the central periodic domain, $1 \leq \mathrm{j} \leq \mathrm{J}$,

$\left\langle K_{\alpha \beta}\right\rangle=\frac{1}{J} \sum_{j=1}^{J} C_{\alpha}(j) C_{\beta}(j)$.

Because for a finite number of particles $\left\langle\mathrm{K}_{\alpha \beta}\right\rangle$ exhibits random noise, we carry out several relaxation runs with different initial configurations, and we average the resulting timehistories. Typically, the amplitude of the noise grows with particle inelasticity.

A numerical scheme based on a fourth-order Runge-Kutta algorithm is used to integrate the ordinary differential Eqs. (4) and (7) for the dilute and dense limits, respectively. First, the algorithm is used to calculate the solution $\mathrm{K}_{\alpha \beta}^{*}$ to the steady problem. Then initial values of the second moments $K_{\alpha \beta}^{1}$ consistent with the imbalance in Eq. (8) are imposed, and the subsequent time-histories are compared with the results of the simulation. For fixed indices $\alpha$ and $\beta$,

$K_{\alpha \beta}^{1}=\xi(\alpha) \xi(\beta) K_{\alpha \beta}^{*}$.

As Figs. (1)-(4) indicate, the theory agrees remarkably well with simulations in the dilute and dense limits for nearly elastic disks. Virtually every feature of the observed timehistories are captured by the theory for all values of $\xi(1)$ and $\xi(2)$ under consideration. 


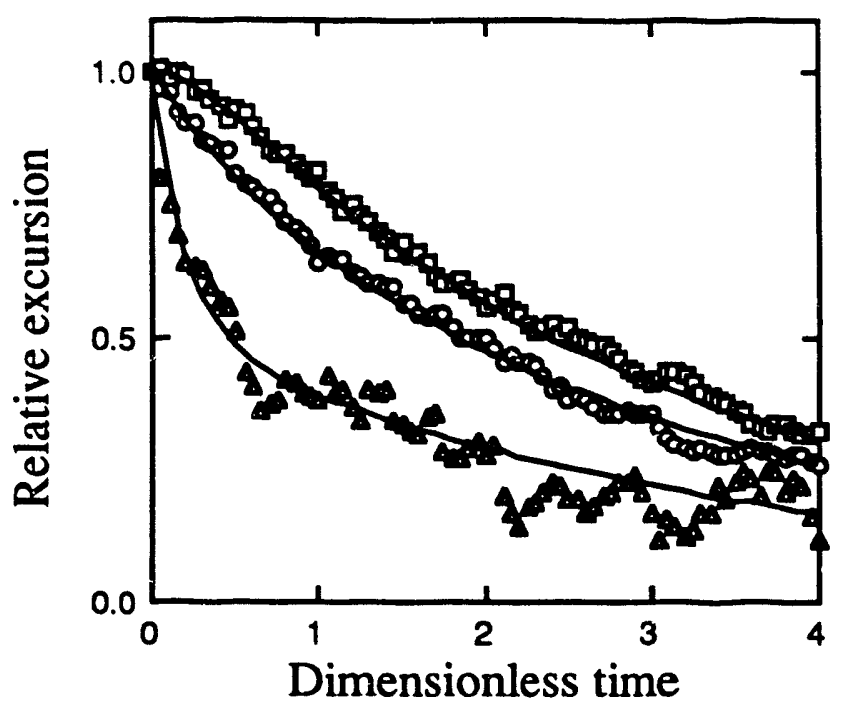

Fig. 1. Dilute, nearly elastic relaxation of $K_{\alpha \beta}$ for the conditions $v=0.1, e=0.9$ and $L / \sigma=83$. Initially, $\xi(1)=\xi(2)=\sqrt{2}$. The abscissa is $\tilde{t}$. For the model, the ordinate is $1-K_{\alpha \beta} / K_{\alpha \beta}^{*}$. For the simulation, it is equal to the average of $1-<K_{\alpha \beta}>/<K_{\alpha \beta}^{0}>$ from ten separate runs. The circles, squares and triangles represent $\left\langle\mathrm{K}_{11}\right\rangle,\left\langle\mathrm{K}_{22}\right\rangle$ and $\left\langle\mathrm{K}_{21}\right\rangle$, respectively. The solid lines are the corresponding predictions of the theory.

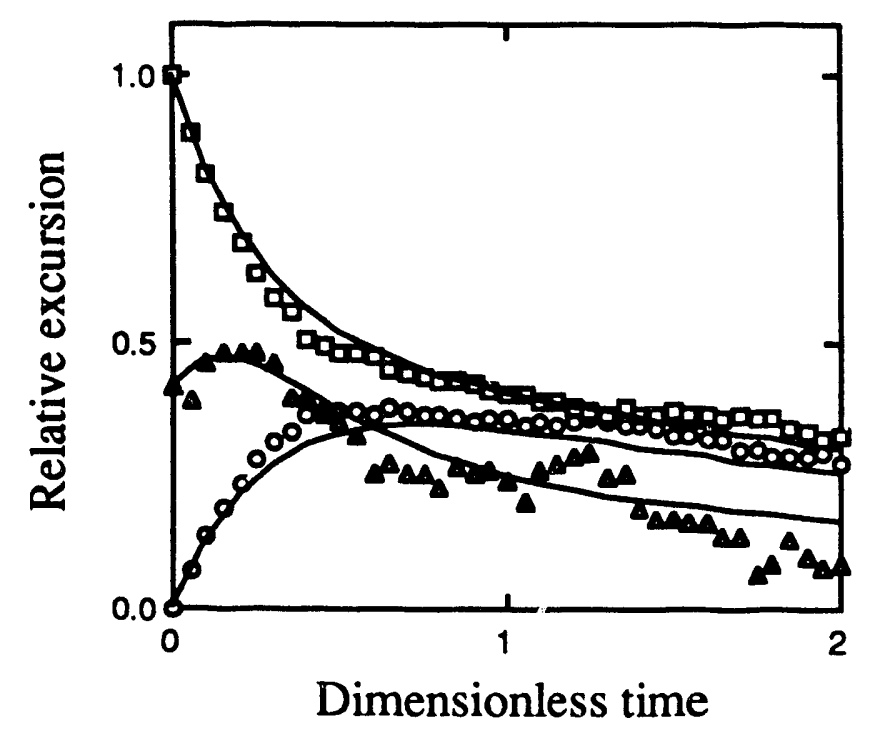

Fig. 2. Dilute, nearly elastic relaxation of $K_{\alpha \beta}$ with $\xi(1)=1, \xi(2)=\sqrt{2}$. See Fig. 1 for conditions and symbols. 


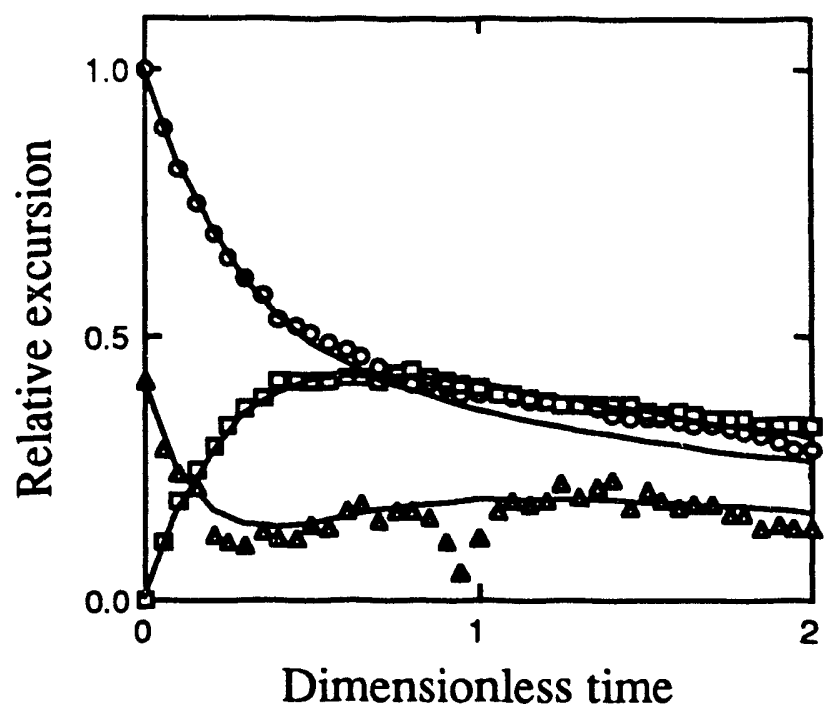

Fig. 3. Dilute, nearly elastic relaxation of $K_{\alpha \beta}$ with $\xi(1)=\sqrt{2}, \xi(2)=1$. See Fig. 1 for conditions and symbols.

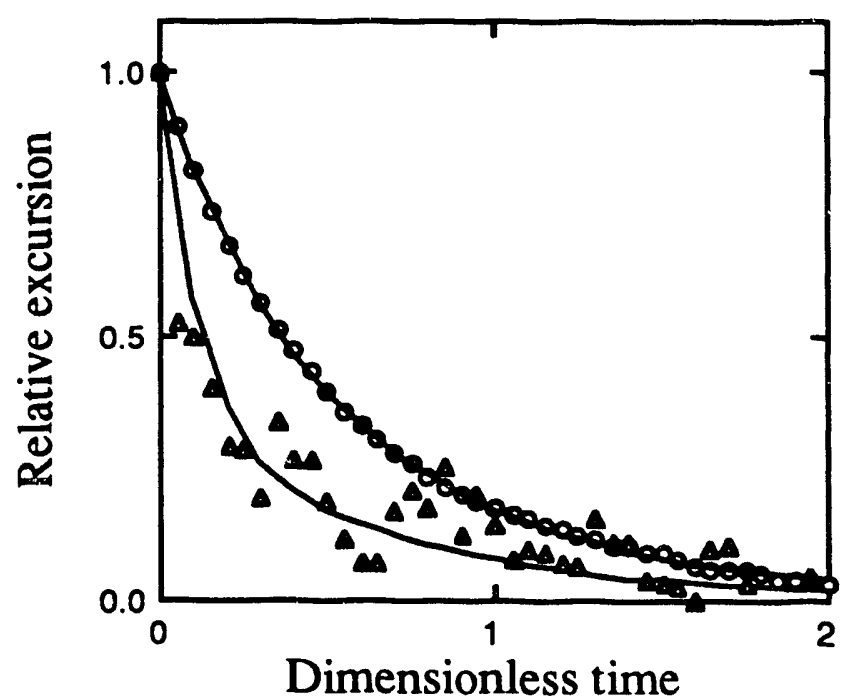

Fig. 4. Dense, nearly elastic relaxation of $T$ and $K_{21}$ for the conditions $v=0.6, e=0.9$ and $L / \sigma=42$ with $\xi(1)=\xi(2)=\sqrt{2}$. The circles represent $1-\langle T>/\langle T O\rangle$ and the triangles $1-\left\langle\mathrm{K}_{21}>\mid<\mathrm{K}_{21}^{0}\right\rangle$ from ten separate runs. The solid lines are the corresponding values of $1-T / T^{*}$ and $1-K_{21} / K_{21}{ }^{*}$ from the theory.

In dilute flows of inelastic particles, the theory agrees well with results of the simulation, although the observed streamwise component $\mathrm{K}_{11}$ consistently lags behind the predictions of the theory (Figs. 5-7). 

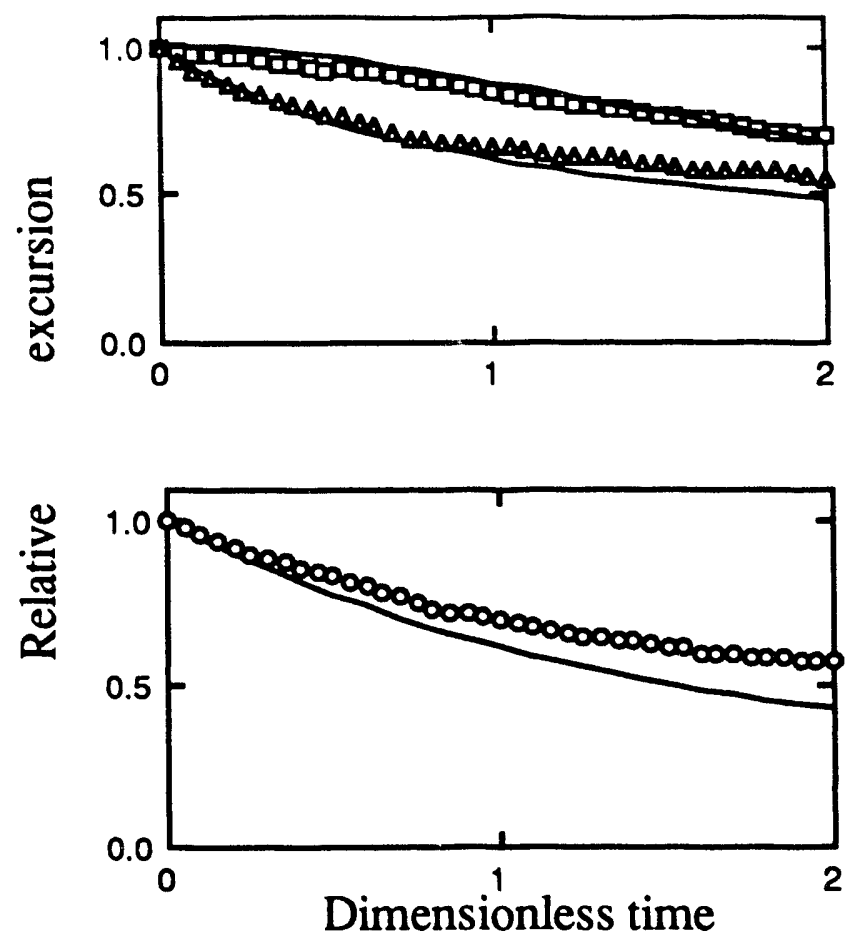

Fig. 5. Dilute, inelastic relaxation of $K_{\alpha \beta}$ for $v=0.1, e=0.2, L / \sigma=83$ and $\xi(1)=\xi(2)=\sqrt{2}$. See Fig. 1 for symbols. Thirty separate runs are averaged.

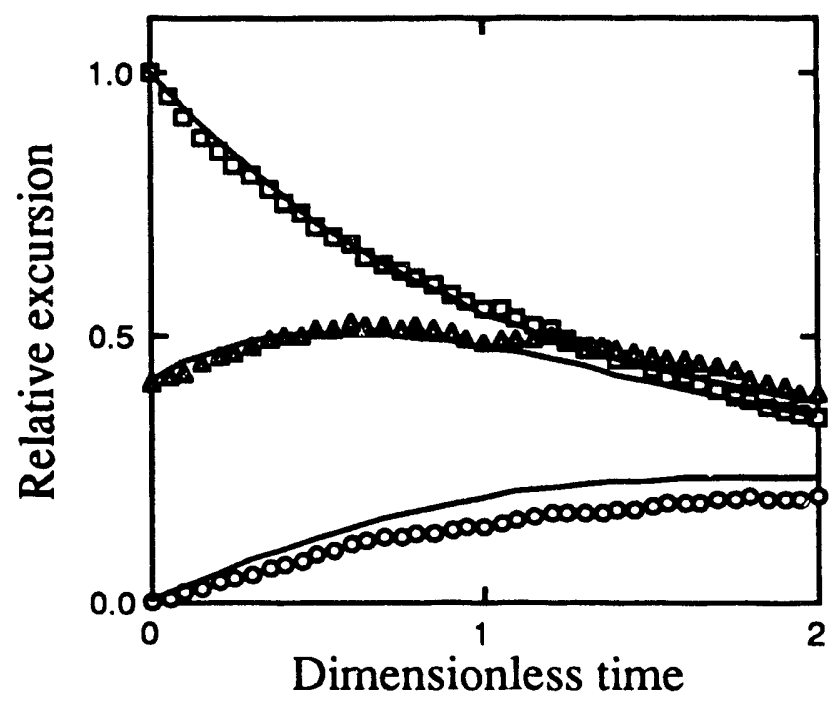

Fig, 6. Dilute, inelastic relaxation of $\mathrm{K}_{\alpha \beta}$ with identical conditions and symbols as Fig. 5 but $\xi(1)=1, \xi(2)=\sqrt{2}$. 


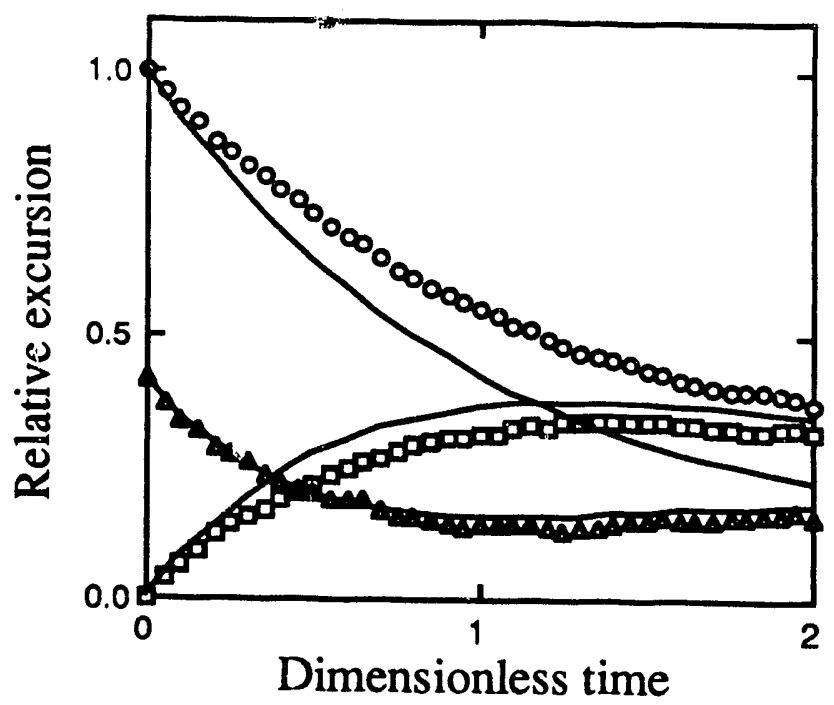

Fig. 7. Dilute, inelastic relaxation of $\mathrm{K}_{\alpha \beta}$ with identical conditions and symbols as Fig. 5 but $\xi(1)=\sqrt{2}, \xi(2)=1$.

In dense flows of inelastic particles, the observed time-histories exhibit a pronounced lag behind the predictions of the theory (Fig. 8). These departures for dense systems of inelastic disks indicate the failure of the assumptions upon which the theory is founded. These include, in order of increasing generality, that the pair distribution function for colliding particles is isotropic, that the velocities of a colliding pair of particles are uncorrelated, that the positions of a colliding pair are not correlated with their velocities, and that the position and time enter into the complete pair distribution function for a colliding pair of particles only through the mean fields of interest and not explicitly. Other evidence of the shortcomings of these assumptions may be found in the substantial deviations between theory and simulation reported by Hopkins and Louge [2] for steady collisional stresses when e $\leq 0.5$ in the dense limit, and in the inelastic microstructure that they describe.

\section{DISCLAIMER}

This report was prepared as an account of work sponsored by an agency of the United States Government. Neither the United States Government nor any agency thereof, nor any of their employees, makes any warranty, express or implied, or assumes any legal liability or responsibility for the accuracy, completeness, or usefulness of any information, apparatus, product, or process disclosed, or represents that its use would not infringe privately owned rights. Reference herein to any specific commercial product, process, or service by trade name, trademark, manufacturer, or otherwise does not necessarily constitute or imply its endorsement, recommendation, or favoring by the United States Government or any agency thereof. The views and opinions of authors expressed herein do not necessarily state or reflect those of the United States Government or any agency thereof. 


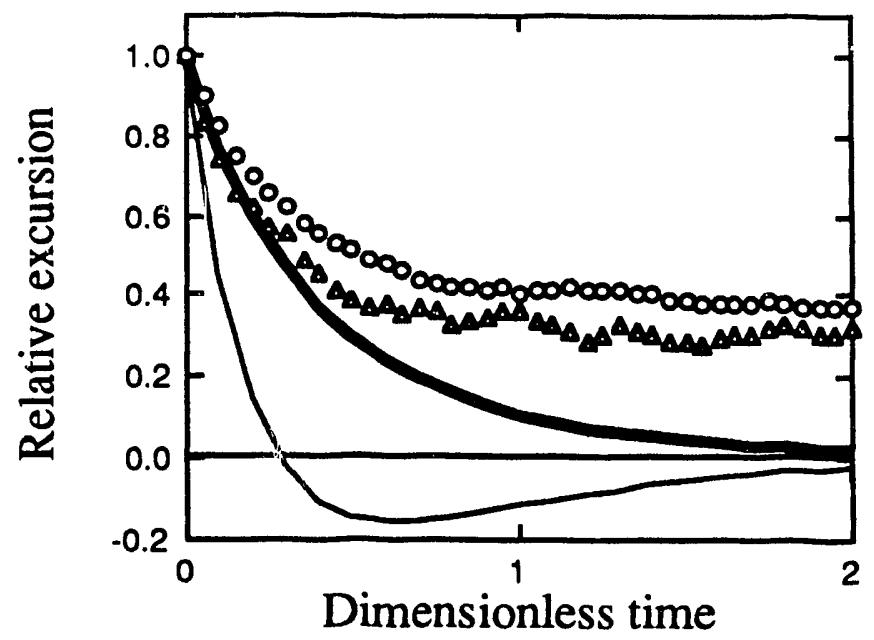

Fig. 8. Dense, inelastic relaxation of $T$ and $K_{21}$ with $v=0.6, e=0.2, L / \sigma=42$ and $\xi(1)=\xi(2)=\sqrt{2}$. See Fig. 4 for symbols. Ten separate runs are averaged. The heavy line represents $1-T / T^{*}$.

\section{Acknowledgements}

This work was supported by the Pittsburgh Energy Technology Center of the US Department of Energy through contracts no DE-AC22-91PC90183 and DE-AC22-88PC88947.

\section{References}

1 J.T. Jenkins and M.W. Richman: "Plane simple shear of smooth inelastic circular disks: the anisotropy of the second moment in the dilute and dense limits," J. Fluid Mech. 192, 313-328 (1988).

2 M.A. Hopkins and M.Y. Louge: "Inelastic microstructure in rapid granular flows of smooth disks," Phys. Fluids A 3 (1), 47-57 (1991). 

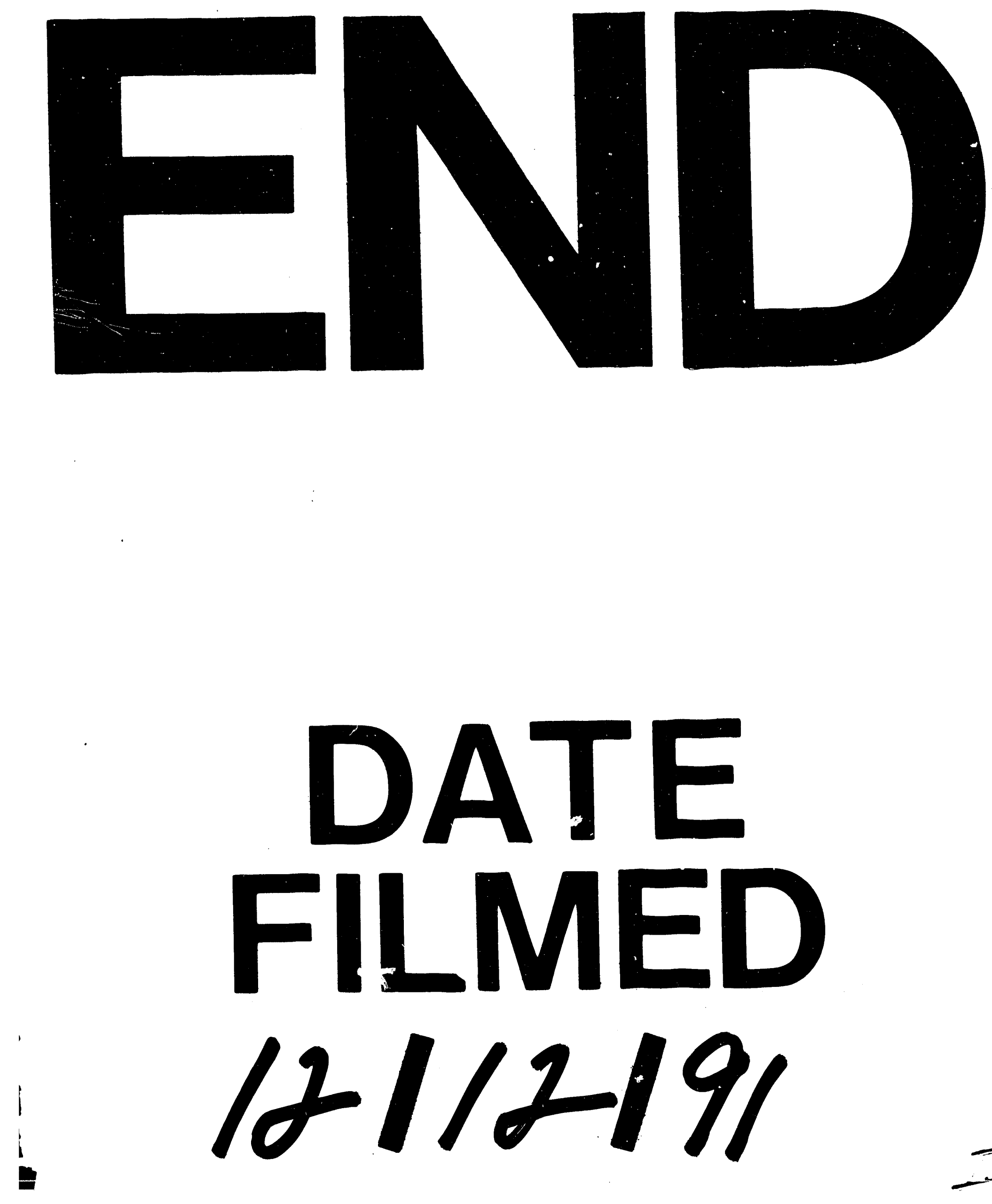
